\title{
THE EFFECT OF POSTERIOR VITREOUS DETACHMENT INDUCED BY INTRAVITREAL INJECTIONS ON MACULAR EDEMA WITH RETINAL VEIN OCCLUSION
}

\author{
Dorukcan Akincioglu ${ }^{1}$, Murat Kucukevcilioglu², Ali Hakan Durukan ${ }^{3}$ \\ ${ }^{1}$ Antalya Ataturk State Hospital, Antalya, Turkey. \\ ${ }^{2}$ Gulhane Training and Research Hospital, Department of Ophthalmology, Ankara, Turkey \\ ${ }^{3}$ University of Health Sciences, Gulhane School of Medicine, Ankara
}

\begin{abstract}
Introduction: To evaluate whether posterior vitreous detachment (PVD) improves treatment outcomes.

Methods: The medical records of treatment-naive patients followed up for a minimum of six months due to retinal vein occlusion between January 2007 and January 2016 were reviewed. Patients with initial PVD were excluded. Correlation analysis and binary logistic regression analysis were used to determine the relationship between categorical variables and treatment outcomes. A subgroup analysis (steroids vs anti-VEGFs) was also performed.
\end{abstract}

Result: The study included 95 eyes of 95 patients with macular edema due to retinal vein occlusion. Patients in both groups [central retinal vein occlusion (CRVO) and branch retinal vein occlusion (BRVO)] were similar in terms of demographic data and showed similar improvement during the study. The patients who underwent intravitreal steroid injection (IVD or IVTA) were 12.35 times (95\% Cl: 4.03-37.85) more likely to develop PVD. The patients in steroid group had a statistically significantly high correlation with visual and anatomic improvements, especially after 5 months of follow-up. The median time of PVD was 5 months (2-11).

Conclusion: Commonly used drugs in intravitreal pharmacotherapy induce PVD, which plays an important role for the treatment of underlying macular edema.

\footnotetext{
Keywords: intravitreal, macular edema, pharmacotherapy, vein occlusion, vitreous detachment

Cite This Article: AKINCIOGLU, Dorukcan; KUCUKEVCILIOGLU, Murat; DURUKAN, Ali Hakan. The effect of posterior vitreous detachment induced by intravitreal injections on macular edema with retinal vein occlusion. International Journal of Retina, [S.I.], v. 4, n. 2, p. 83, sep. 2021. ISSN 2614-8536. Available at: $<$ https://www.ijretina.com/index.php/ijretina/article/view/162>.doi: https://doi.org/10.35479/ijretina.2021.v ol004.iss002.162.
}

Correspondence to:

Dorukcan Akincioglu,

Antalya Ataturk State Hospital,

Antalya, Turkey,

dr.dorukcan@yahoo.com

\section{INTRODUCTION}

Visual complaints due to retinal vein occlusion (RVO) are commonly caused by macular edema (ME). ${ }^{1}$ Before the era of intravitreal pharmacotherapy, laser photocoagulation was the only treatment modality for ME secondary to RVO. The central vein occlusion study (CVOS) showed that grid photocoagulation did not improve visual outcomes; however, ME was reduced. ${ }^{2}$ After this study, observation has become the standard care for the treatment of ME following central retinal vein occlusion (CRVO). 
On the other hand, macular grid laser remains the standard of care at that time for the treatment of ME following branch retinal vein occlusion (BRVO). ${ }^{3}$ The standard care vs. corticosteroid for retinal vein occlusion (SCORE) study showed that patients in the intravitreal corticosteroid treatment groups were five times more likely to have visual gain in one year. ${ }^{4}$ However, those who received $4 \mathrm{mg}$ dose had the highest rates of ocular side effects. ${ }^{4}$ These results of intravitreal steroid therapy for RVO constituted a major milestone for patients. Following this study, clinical trials, such as like COPERNICUS ${ }^{5}$, VIBRANT $^{6}$, and RETAIN $^{7}$ reported that agents acting through vascular endothelial growth factor (VEGF) were also effective in the treatment of ME caused by RVO and they had less frequent ocular side effects.

In the literature, it is reported that the posterior vitreous cortex plays an important role in the exacerbation of macular edema in retinal diseases. ${ }^{8}$ However, none of the previous clinical studies analyzed the role of PVD following intravitreal injections in patients with RVO alone. The primary goal of this study was to evaluate whether posterior vitreous detachment (PVD) was correlated with visual prognosis, and whether there were significant differences between intravitreal agents in inducing PVD. Our secondary goal was to report our results and compare them to randomized clinical trials on RVO therapy.

\section{METHODS}

This study was a retrospective, interventional, comparative case series of treatment-naive patients with ME secondary to RVO followed up at the retina center of "..."Training and Research Hospital from January 2007 to May 2016. The study protocol was approved by the Institutional Review Board of "..." Medical School and followed the tenets of the Declaration of Helsinki.
The medical records and images of 212 patients were reviewed, and 95 eyes of 95 eligible patients with ME secondary to RVO were included in the study. The patients had previously received no treatment for RVO, their central macular thickness (CMT) was above $250 \mu \mathrm{m}$ and visual acuity was worse than 0.1 LogMAR, and they had at least six months' follow-up. The exclusion criteria were the presence of PVD, having insufficient medical data, having a history of vitreoretinal surgery or concomitant ocular diseases (glaucoma, vitreous hemorrhage, diabetic retinopathy, uveitis, vitreoretinal interface pathologies, age-related macular degeneration, and retinal arterial occlusion), and having lenticular/corneal opacity that could interfere with detailed optic coherence tomography (OCT) and fundus fluorescein angiography (FFA) examinations. Data, including previous eye disease and systemic diseases, such as diabetes mellitus and systemic hypertension were collected at the first visit. Each ophthalmic examination included the evaluation of best-corrected visual acuity (BCVA) in Snellen decimal units (converted to LogMAR units), slit-lamp examination, dilated fundus examination, intraocular pressure (IOP), and spectral-domain OCT (Heidelberg Spectralis SD-OCT; Heidelberg Engineering, Inc., Heidelberg, Germany). The examinations were performed at the first visit, third month, sixth month, and 12th month. Posterior segment photography and FFA with the Heidelberg retinal angiography (HRA-2; Heidelberg Engineering, Inc., Heidelberg, Germany) were performed either at the first visit or later when retinal hemorrhage was resolved to a level that allowed obtaining clear images for the evaluation of ischemia. PVD was considered to be present if Weiss' ring was observed during the fundus examination, posterior vitreous hyaloid is showed no connection to the optic nerve head in ultrasound-B examination or posterior vitreous membrane was observed above the retina during the OCT examination. ${ }^{9}$ 
Patients with BRVO were evaluated as ischemic if they had capillary perfusion defects larger than 5 optic disc diameters at presentation. ${ }^{10}$ In the CRVO group, the patients were evaluated as ischemic if they had capillary perfusion defects larger than 10 optic disc diameters. ${ }^{11}$ Our patients had five different intravitreal pharmacotherapies based on the treatment plan: intravitreal dexamethasone (IVD, Ozurdex ${ }^{\circledR}$, Allergan plc, Dublin, Ireland), intravitreal triamcinolone acetonide (IVTA, $4 \mathrm{mg} / \mathrm{mL}$-Kenacort ${ }^{\circledR}$ A40; Bristol-Myers Squibb, New York, NY, USA), intravitreal bevacizumab (IVB, 1,25mg/0,05mLAvastin ${ }^{\circledR}$; Genentech, Inc., South San Francisco, CA, USA), intravitreal ranibizumab (IVR, 0,5mg/0,05mLLucentis ${ }^{\circledR}$; Genentech, South San Francisco, CA, USA, and Novartis Pharma AG, Basel, Switzerland), and intravitreal aflibercept (IVA, 2mg/0,05mLEYLEA ${ }^{\circledR} ;$ Bayer Health Care AG, Berlin, Germany). The BRVO group did not receive aflibercept treatment due to the national health insurance policy at the time of the study. According to the pharmacotherapy applied, the patients were evaluated in two main groups as the steroid group (IVD + IVTA) and the anti-VEGF group (IVB + IVR + IVA).

We performed retreatment on an as-needed basis. The retreatment criteria were $a \geq 1$ line decline in BCVA and a CFT of $\geq 300 \mu \mathrm{m}$ at the follow-up visits. For the patients that did not need retreatment at the first follow-up visit, the minimum interval for the next visit was six weeks for the IVB, IVR and IVA groups, and 12 weeks for the IVD group. CMT was determined as the distance between the inner limiting membrane and the retinal pigment epithelium within an area of $1 \mathrm{~mm} 2$ in the central region. Central macular volume (CMV) was measured using the ETDRS plot of macular topography. The absolute change in CMT (ACCMT) was determined as the difference between the pretreatment CMT and the CMT value obtained at the following month after treatment. Similarly, the absolute change in CMV (ACCMV) was determined as the difference between the pretreatment CMV and the CMV value obtained at the following month after treatment.

In statistical analysis, the normality of distribution was checked using the Shapiro-Wilk test. The chisquare test or Fisher's exact test was used for discrete variables. The Mann-Whitney $U$ test was conducted for continuous variables. The Wilcoxon test was used to compare dependent groups. The Spearman rank correlation test was used for correlation analysis. The binary logistic regression analysis was undertaken to test categorical variables. Statistical analyses were performed using SPSS software for Windows (v. 21.0, Chicago, IL).

Ninety-five eyes of 95 patients with ME secondary to RVO were included in the study. Forty-eight (50.5\%) of the patients had BRVO, and 47 (49.5\%) had CRVO. The demographic and clinical characteristics of the patients were similar between the CRVO and BRVO treatment groups (Table-1).

\section{RESULTS}

Ninety-five eyes of 95 patients with ME secondary to RVO were included in the study. Forty-eight (50.5\%) of the patients had BRVO, and 47 (49.5\%) had CRVO. The demographic and clinical characteristics of the patients were similar between the CRVO and BRVO treatment groups (Table-1). 


\begin{tabular}{lcc}
\hline \multicolumn{3}{l}{ Table-1: Baseline demographics of the patients } \\
\hline & Steroid group & Anti-VEGF group \\
CRVO & $13(32.5 \%)$ & $34(61.8 \%)$ \\
BRVO & $27(67.5 \%)$ & $21(38.2 \%)$ \\
Female & $15(37.5 \%)$ & $17(30.9 \%)$ \\
Male & $25(62.5 \%)$ & $38(69.1 \%)$ \\
Non-ischemic & $28(50.9 \%)$ & $20(50 \%)$ \\
Ischemic & $27(49.1 \%)$ & $20(50 \%)$ \\
Age (Mean \pm SD) & $61.08 \pm 13.44$ & $58.58 \pm 14.17$ \\
Pretreatment BCVA & $0.85 \pm 0.69$ & $1.12 \pm 0.80$ \\
Pretreatment CMT & $629.45 \pm 256.53$ & $716.20 \pm 260.64$ \\
Pretreatment CMV & $0.46 \pm 0.15$ & $0.50 \pm 0.14$ \\
Injection number* & $1.38 \pm 0.71$ & $2.56 \pm 1.28$ \\
(Mean \pm SD) & & \\
\hline
\end{tabular}

Both disorder groups showed statistically significant functional and anatomic improvement for the steroid and anti-VEG subgroups $(p<0.05)$. The largest and significantly longest visual gain during the follow-ups was observed in the IVB subgroup. However, visual and anatomic improvement did not statistically significantly differ between the steroid and anti-VEGF groups (Fig-1)

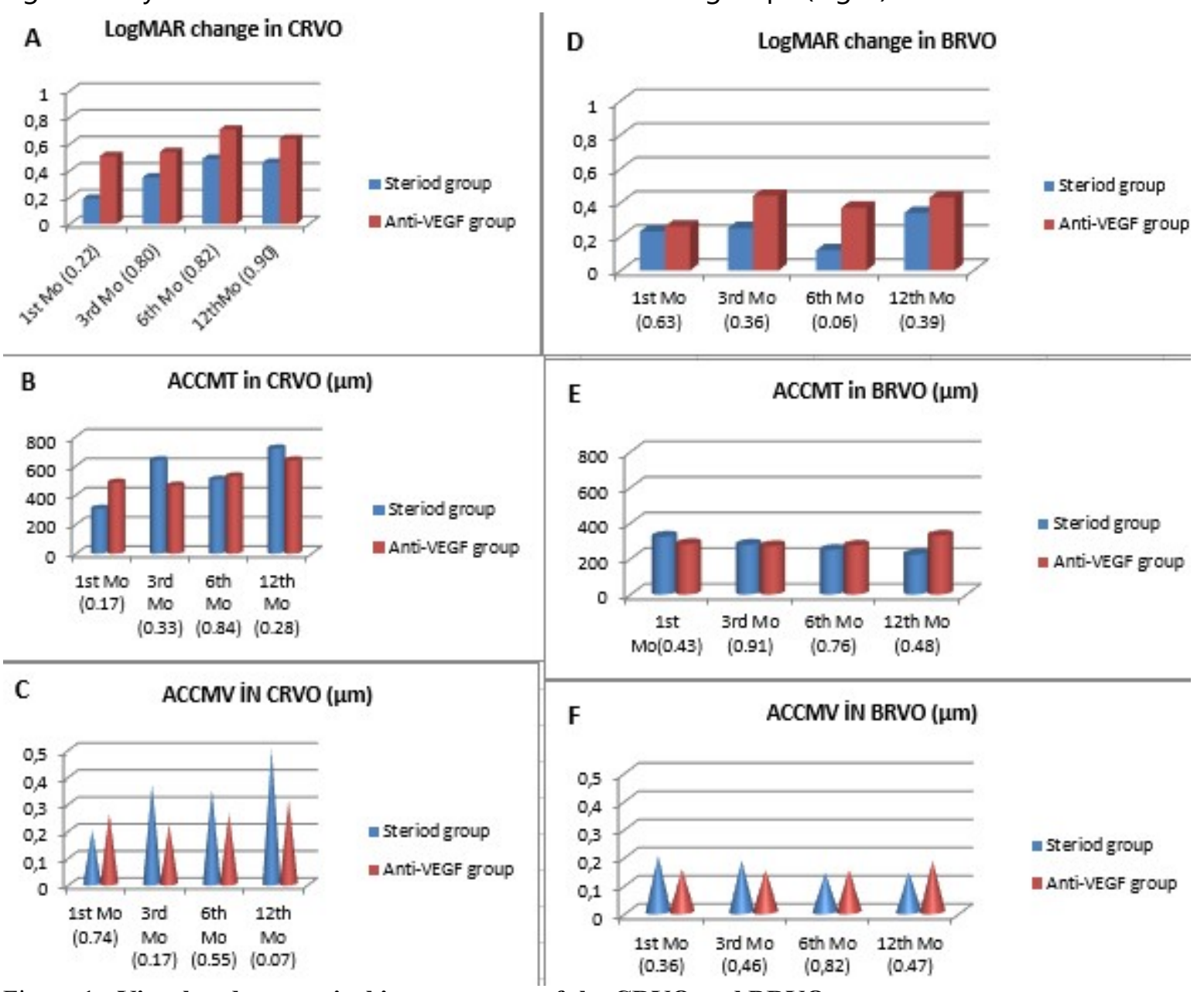

Figure-1: Visual and anatomical improvement of the CRVO and BRVO groups. 
A: LogMAR changes in the treatment groups for the patients with CRVO over 12 months, and the corresponding $p$ values are given.

$B$ : Absolute change in the central macular thickness (ACCMT) for the CRVO group and the corresponding $p$ values are given.

$C$ : Absolute change in the central macular volume (ACCMV) for the CRVO group and the corresponding $p$ values are given.

D: LogMAR changes in the treatment groups for the patients with BRVO over 12 months and the corresponding $p$ values are given.

$E: A C C M T$ in the treatment groups for the patients with BRVO over 12 months and the corresponding $p$ values are given.

F: ACCMV changes in the treatment groups for the patients with BRVO over 12 months and the corresponding $p$ values are given.

Eight (23.5\%) patients with CRVO in the anti-VEGF group had to be switched to another agent due to retreatment criteria. The new agent was mostly a dexamethasone implant (62.5\%). PVD was observed in four $(11.8 \%)$ and eight (61.5\%) eyes in the antiVEGF and steroid subgroups, respectively. In addition, four eyes developed PVD after switching to a dexamethasone implant. Eighty-six percent of the patients that initially received IVTA needed to be switched to other agents due to ocular side effects.
PVD after switching to a dexamethasone implant. Sixty-five percent of the cases in the BRVO group that initially had IVTA needed to be switched to other agents due to ocular side effects.

Patients who underwent intravitreal steroid injections (IVD and IVT) were 12.35 times $(95 \% \mathrm{Cl}$ : 4.03-37.85) more likely to develop PVD when compared to those that received intravitreal antiVEGF injections (IVB, IVR and IVA). There were no

\begin{tabular}{lcccccc}
\hline \multicolumn{6}{l}{ Table-2. Results of the correlation analysis between visual and anatomical improvement } \\
\hline Visual & Third-month & Sixth-month & $12^{\text {th }}$ - & Third-month & Sixth-month & $12^{\text {th }}$-month \\
improvemen & CMT & CMT & monthCMT & CMV & CMV & CMV \\
$t$ & improvemen & improvemen & improvemen & improvemen & improvemen & improvemen \\
& $t(\%)$ & $t(\%)$ & $t(\%)$ & $t(\%)$ & $t(\%)$ & $t(\%)$
\end{tabular}

Anti-VEGF

Group ( $\mathbf{r} ; \mathbf{p})$

$\begin{array}{llll}\text { Third month } & -0.28 ; 0.92 \quad-\quad & - & -0.32 ; 0.05\end{array}$

$\begin{array}{lcccccc}\text { Sixth month } & - & -0.17 ; 0.73 & - & - & -0.26 ; 0.96 & \\ \text { 12th month } & - & - & -0.24 ; 0.62 & - & - & -0.37 ; 0.81 \\ \text { Steroid } & & & & & & \\ \text { Group (r; p) } & & & & & & \\ \text { Third month } & -0.37 ; 0.06 & - & - & -0.30 ; 0.13 & - & - \\ \text { Sixth month } & - & -0.61 ; 0.009 & - & - & -0.71 ;<0.001 & -\end{array}$

In the BRVO group, 11 (52.4\%) patients in the antiVEGF group required switching to another agent due to retreatment criteria. Similar to the CRVO group, the new agent was mostly a dexamethasone implant (42.9\%). PVD was observed in one (4.8\%) and 14 (51.9\%) eyes in the anti-VEGF and steroid subgroups, respectively. Furthermore, seven patients developed statistically significant differences in terms of functional and anatomical improvement between the eyes with and without PVD. However, a statistically significant high correlation was observed between visual improvement and percent change of foveal thickness at the third, sixth and 12th months (Table-2). 
In our study, PVD development was observed with a median time of 5 (1-11) months. The correlation analysis revealed a statistically significant correlation in the steroid group by the sixth month. The binary logistic regression analysis revealed that categorical variables, namely ischemia ( $p=0.56$ ), hypertension $(p=0.84)$, and diabetes $(p=0.68)$ were not significantly correlated with a reduction of $>0.25$ $\mathrm{mm} 3$ in CMV. The only categorical variable statistically significantly correlated with $\mathrm{CMV}$ was was $27.4 \%$, and the mean age for this population was $61.62 \pm 13.22$ years. The PVD percentages in patients having anti-VEGF injections were previously reported as $24 \%$ over an 11.1 -week (mean) period. ${ }^{13}$ In our study, 55 patients were followed up after one or more intravitreal anti-VEGF injections over 12 months during which five eyes (9.1\%) developed PVD. Alpay reported a rate of $26.9 \%$ for the PVD development following dexamethasone-implant injections. ${ }^{14}$ We observed similar (27.4\%) rates of
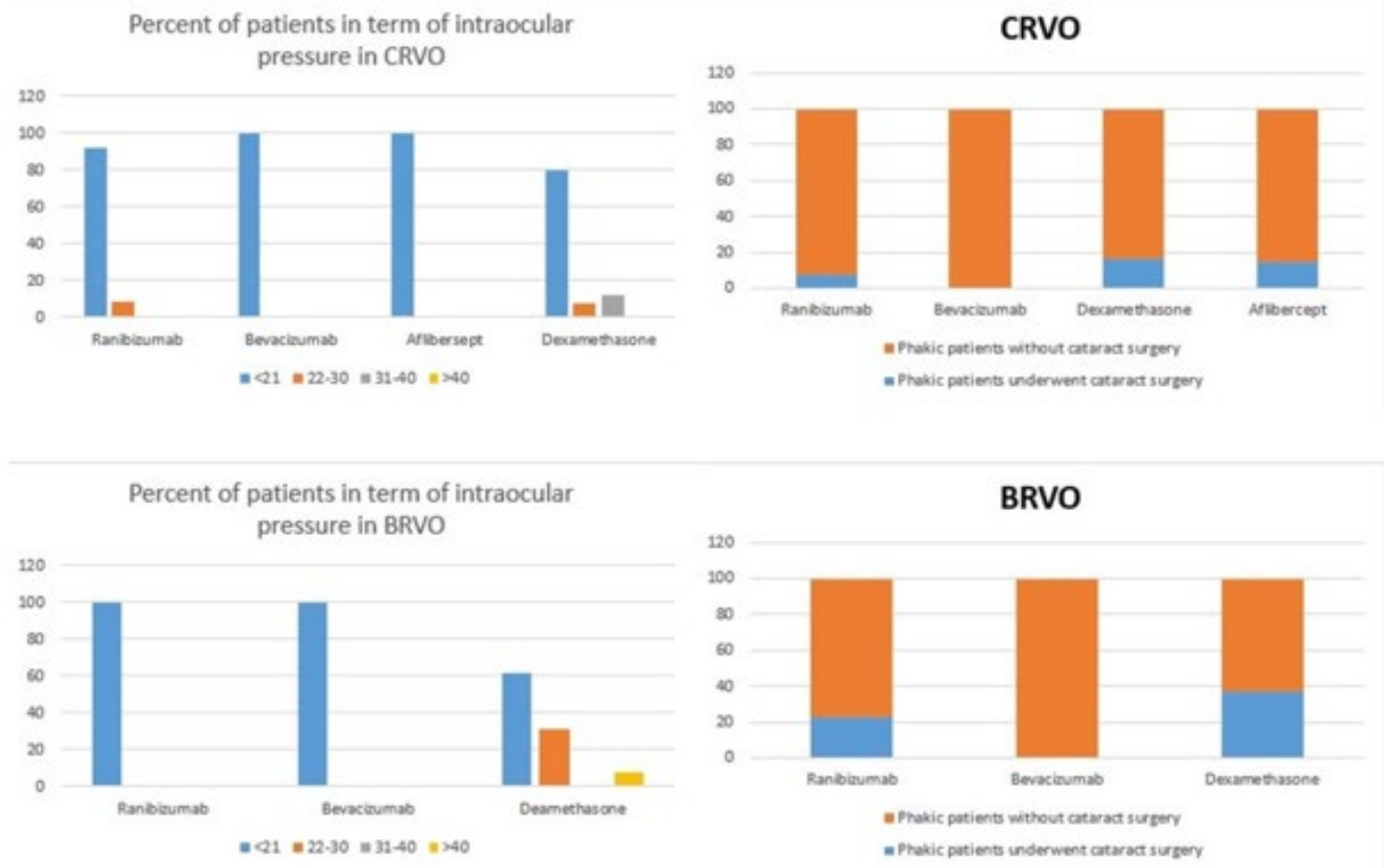

Figure 2. Percentages of patients that developed ocular side effects during intravitreal pharmacotherapy

PVD ( $p=0.033)$. In addition, a decrease of more than $50 \%$ in CMT compared to the baseline was present in $6 \%$ of the patients without PVD and $15 \%$ of those with PVD. The rates of a macular volume decrease of more than $50 \%$ compared to the baseline were $12 \%$ and $22.8 \%$ for the eyes with posterior vitreous attachment and PVD, respectively. Ocular side effects are shown in Figure-2.

\section{DISCUSSION}

Weber-Krause et al. $^{12}$ reported that the PVD ratio increased with aging, ranging from $27 \%$ to $61 \%$ in different age groups (60 to 90 years). In our study, we excluded patients presenting with PVD before treatment, and the mean age was $59.63 \pm 13.85$ years in our population. The posttreatment PVD rate
PVD following dexamethasone -implant injections, but both of the previously mentioned studies included patients with heterogenous retinal diseases, such as RVO, age-related macular degeneration, and diabetic macular edema whereas we only evaluated RVO cases. This may be significant since each retinal pathology may affect the vitreoretinal interface differently. Interestingly, in a phase III multicenter clinical trial, Stalmans et al. reported that $26.5 \%$ of ocriplasmin-injected eyes and $10.1 \%$ of placebo-injected eyes developed PVD. ${ }^{15}$ The cause of PVD might be the mechanic effect of drug volume, but one study reported that none of the patients treated with a combination of bevacizumab and triamcinolone developed 
PVD despite a higher volume of drug injection into the vitreous while $28 \%$ of patients treated with bevacizumab alone developed PVD. ${ }^{13}$

Randomized clinical trials focus on vascular extravasation by blocking receptors, not through the mechanical effect of taut or PVD. In the CRUISE study, eyes with ME due to CRVO were treated with monthly intravitreal ranibizumab injections ${ }^{16}$. In that study, the patients received six monthly injections, followed by further injections as needed. The average visual gain was 13.9 letters at the 12thmonth follow-up examination. However, the CRUISE study did not evaluate patients with ischemic CRVO. In our CRVO group, patients with ischemic CRVO were not excluded. In our IVR group, the baseline visual acuity was $1.12 \pm 0.82$ LogMAR, and the average visual acuity was $1.05 \pm 1.48$ LogMAR at the 12th-month follow-up examination after a mean of two (1-6) injections. The improvement in the OCT parameters was statistically significant for nine months. In the CRUISE study, the mean change from the baseline CMT was a reduction of $452.3 \mu \mathrm{m}$ at the sixth month after monthly injections. This reduction was maintained through as-needed injections, and the mean reduction from the baseline CMT was $462.1 \mu \mathrm{m}$ at the 12 th month. ${ }^{17}$ In our study, CMT was reduced by $612 \mu \mathrm{m}$ and $736.3 \mu \mathrm{m}$ in the IVR group for CRVO at the sixth and 12th months, respectively. Our patients had a mean of 2.75 injections over 12 months; however, the patients in the CRUISE group had six monthly injections, followed by an average number of 3.6 injections as needed for the sixth to 12th months. We consider that our anatomical results in the CRVO group were similar, even with much fewer injections. On the other hand, we are aware that the half-life of ranibizumab is below three days in animal studies ${ }^{18}$, and around nine days in human vitreus. ${ }^{19}$ Therefore, some investigators suggest that patients that have completed a threemonth loading phase with monthly injections achieve earlier and better visual improvement. ${ }^{20}$
In the BRAVO study, eyes with ME due to BRVO were administered six monthly injections first, and then further injections as needed. The visual gain was 18.3 letters at the 12th-month follow-up examination. In our BRVO group, the baseline visual acuity was $0.66 \pm 0.49$ LogMAR, and the average visual acuity was $0.30 \pm 0.14$ LogMAR at the 12thmonth follow-up examination, with a mean of two (1-5) injections. In our study, visual acuity grading was evaluated in LogMAR, but in both CRUISE and BRAVO studies, visual improvement was evaluated based on letters. Therefore, we cannot compare our results on visual improvement to these studies. In the BRAVO study, the mean change from the baseline CMT was a reduction of $345 \mu \mathrm{m}$ at the sixth month after monthly injections. These reductions were maintained with as-needed injections with a mean reduction of $347.4 \mu \mathrm{m}$ in CMT at the 12th month compared to the baseline. In our study, the reduction in CMT was $380.2 \mu \mathrm{m}$ and $400.6 \mu \mathrm{m}$ in the IVR group for BRVO at the sixth and 12th months, respectively. Our patients had a mean of 2.2 injections over 12 months; however, the patients in the BRAVO study had six monthly injections, followed by an average of 2.8 injections as needed.

To date, no well-designed randomized controlled trial has been undertaken to establish the efficacy and safety of intraocular bevacizumab. Nevertheless, there is a widespread off-label use of bevacizumab in clinical ophthalmology. Yuan et al. ${ }^{21}$ concluded that both ranibizumab and bevacizumab were effective for the treatment of RVO and appeared to have similar visual and anatomical outcomes. In our study, the eyes in the IVB group had significant improvement in terms of visual and anatomical outcomes for the first 10 months compared to pretreatment. There were no patients that underwent cataract surgery in the IVB group during the follow-up, although all eyes were phakic. The efficacy of bevacizumab in patients with CRVO is reported to be around two months. ${ }^{22}$ In our study, the patients in the IVB group received a mean of two (1-6) injections and had better visual and anatomical 
improvements. Nevertheless, this improvement was not statistically significant when compared to the remaining treatment subgroups. Repeated injections are needed in most eyes for the continued control of ME and preserved visual improvement. ${ }^{22}$ Our patients in the IVB group had longer anatomical and visual improvement in correlation with the number of injections.

Aflibercept is the only anti-VEGF agent known to have efficacy in ischemic CRVO.5 In studies, such as COPERNICUS and GALILEO, the baseline perfusion status was evaluated, and both patients with perfused retinas and those with non-perfused retinas treated with intravitreal aflibercept experienced similar visual improvement. The subgroup analysis of our patients in the IVA group also showed that those with perfused and nonperfused CRVO had similar anatomical and visual improvements. Although the level of improvement in the IVA group in our study was not as high as reported by the COPERNICUS and GALILEO studies, the percentages obtained from the ischemic and non-ischemic patients were similar. On the other hand, these two previous studies did not have any data concerning baseline OCT parameters, which may have caused this difference. In terms of ACCMT alone, this value was $450 \mu \mathrm{m}$ in both previous randomized clinical trials in contrast to our study, in which ACCMT was calculated as $263 \mu \mathrm{m}$ at the sixth month. We consider that this difference may result from the unequal number of injections and significantly different baseline CMTs.

The GENEVA trial had an unusual design that included both CRVO and BRVO patients treated with dexamethasone implants. ${ }^{23}$ For all eyes, the mean decrease in CMT was significant with dexamethasone implants of $0.7 \mathrm{mg}(208 \pm 201 \mu \mathrm{m})$ and $0.35 \mathrm{mg}(177 \pm 197 \mu \mathrm{m})$ compared with sham treatment $(85 \pm 173 \mu \mathrm{m})$ at the third month but not at the sixth month. In our study, both CRVO and BRVO cases had significant improvement, whether ischemic or not, for six months, and ACCMT was 540 $\mu \mathrm{m}$ and $242 \mu \mathrm{m}$ for the CRVO and BRVO groups, respectively. The differences between the groups were related to the baseline CMT differences. We observed that improvement remained at a significant level for the first four months, after which it began to decrease. The cataract progression rates were $29.8 \%$ in the GENEVA trial and $33.3 \%$ in our study.

Being a retrospective study, this study had the limitation of not being able to evaluate cataract grading. We did not know baseline cataract grades in any group, and there was no cataract grading during the follow-up. The cataract progression rates in our study indicate that the patients underwent cataract surgery during routine follow-up examinations, and this was the highest in the IVD group. In addition, the IVR and IVA groups presented good outcomes anatomically but not visually due to the high cataract progression rates in these groups. As previously mentioned, this data is not sufficient to support the idea that IVR and IVA result in higher rates of ocular complications compared to IVB due to the retrospective nature and lack of cataract grading during the follow-up. Furthermore, cataract extraction can promote PVD, but there is no way to ascertain the exact reason. Vitreous volume and size of the eye play an important role in the development of PVD. Axial length measurement is very important for determining the effect of eye size on the rate of PVD. When we evaluated the patients without previous glaucoma, the highest rate of those with increased intraocular pressure were found in the IVTA group (20\%). We did not see any retinal detachment or endophthalmitis during the followup period in any of our groups.

\section{CONCLUSIONS}

As a result of our study, we suggest that bevacizumab can be used for the treatment of $\mathrm{ME}$ due to RVO. If this is not an option, being an off-label drug, ranibizumab may be an alternative. The risk of contamination is high while aspirating the required dose from the bevacizumab vial multiple times or preparing multiple vials from the original vial when 
compared to the use of prefilled syringes. A dexamethasone implant may be a choice in pseudophakic patients with no known history of glaucoma. An intravitreal injection itself can induce PVD, which was mostly due to dexamethasone implants in our study. PVD potentially has a positive effect on the underlying macular disease with high rates of decreased macular volume.

Declaration of interest statement: The authors report no conflict of interest.

Data availability: Data available on request due to privacy/ethical restrictions

\section{REFERENCES}

1. Karia N. Retinal vein occlusion: pathophysiology and treatment options. Clin Ophthalmol. 2010;4:809-16

2. The Central Vein Occlusion Study Group. Evaluation of grid pattern photocoagulation for macular edema in central vein occlusion. Ophthalmology 1995; 102:1425-33 DOI: 10.1016/s0161-6420(95)30849-4

3. The Branch Vein Occlusion Study Group. Argon laser photocoagulation for macular edema in branch vein occlusion. Am J Ophthalmol.1984;98(3):271-82

4. Ip MS, Scott IU, VanVeldhuisen PC, Oden NL, Blodi BA, Fisher $M$ et al. SCORE Study Research Group. A randomized trial comparing the efficacy and safety of intravitreal triamcinolone with observation to treat vision loss associated with macular edema secondary to central retinal vein occlusion: the Standard Care vs Corticosteroid for Retinal Vein Occlusion (SCORE) study report 5.Arch Ophthalmol.,2009;127(9):1101-14

5. Heier JS, Clark WL, Boyer DS, Brown DM, Vitti R, Berliner AJ et al. Intravitreal aflibercept injection for macular edema due to central retinal vein occlusion: two-year results from the COPERNICUS study. Ophthalmology 2014;121(7):1414-20
6. Campochiaro PA, Clark WL, Boyer DS, Heier JS, Brown DM, Vitti R et al. Intravitreal aflibercept for macular edema following branch retinal vein occlusion: the 24-week results of the VIBRANT study. Ophthalmology.2015;122(3):538-44

7. Campochiaro PA, Sophie R, Pearlman J, Brown DM, Boyer DS, Heier JS et al. RETAIN Study Group. Long-term outcomes in patients with retinal vein occlusion treated with ranibizumab: the RETAIN study Ophthalmology,2014;121:209-19

8. Jackson TL, Nicod E, Angelis A, Grimaccia F, Prevost AT, Simpson AR et al Vitreous attachment in age-related macular degeneration, diabetic macular edema, and retinal vein occlusion: a systematic review and metaanalysis. Retina , 2013;33:1099-1108.

9. Bertelmann T, Goos C, Sekundo W, Schulze S, Mennel S. Is optical coherence tomography a useful tool to objectively detect actual posterior vitreous adhesion status? Case Rep Ophthalmol Med. 2016;2016:3953147.

10. Branch Vein Occlusion Study Group. Argon laser scatter photocoagulation for prevention of neovascularization and vitreous hemorrhage in branch vein occlusion. A randomized clinical trial.Arch Ophthalmol.1986;104(1):34-41

11. Central Retinal Vein Occlusion Study Group. Natural history and clinical management of central retinal vein occlusion. Arch Ophthalmol.,1997;115: 486-91

12. Weber-Krause $B$, Eckardt $U$. Incidence of posterior vitreous detachment in eyes with and without age-related macular degeneration. An ultrasonic study. Ophthalmologe. 1996;93(6):660-665.

13- Geck U, Pustolla N, Baraki H, Atili A, Feltgen N, Hoerauf $H$. Posterior vitreous detachment following intravitreal drug injection. Graefes Arch Clin Exp Ophthalmol. 2013;251(7):16911695 
14. Alpay A. Posterior vitreous detachment rate following intravitreal dexamethasone injection. Int J Ophthalmol. 2019;12(8):1298-1303. Published $2019 \quad$ Aug 18. doi:10.18240/ijo.2019.08.10

15. Stalmans P, Benz MS, Gandorfer A, Kampik A, Girach A, Pakola $S$ et al. MIVI-TRUST Study Group Enzymatic vitreolysis with ocriplasmin for vitreomacular traction and macular holes. $\mathrm{N}$ Engl J Med, 2012;367:606-615

16. Campochiaro PA, Brown DM, Awh CC, Lee SY, Gray $S$, Saroj $N$ et al. Sustained benefits from ranibizumab for macular edema following central retinal vein occlusion: twelve-month outcomes of a phase III study. Ophthalmology.2011;118(10):2041-49

17. Brown DM, Campochiaro PA, Bhisitkul RB, Ho AC, Gray $S$, Saroj $N$ et al. Sustained benefits from ranibizumab for macular edema following branch retinal vein occlusion: twelve-month outcomes of a phase III study. Ophthalmology.2011;118(8):1594-602

18. Bakri SJ, Snyder MR, Reid JM, Pulido JS, Ezzat MK, Singh RJ et al. Pharmacokinetics of intravitreal ranibizumab (Lucentis). Ophthalmology. 2007;114(12):2179-82

19. Xu L, Lu T, Tuomi L, Jumbe N, Lu J, Eppler $S$ et al. Pharmacokinetics of ranibizumab in patients with neovascular age-related macular degeneration: a population approach. Invest Ophthalmol Vis Sci. 2013;54(3):1616-24

20. Lucentis (ranibizumab) injection [prescribing information revised] Genentech, Inc, South San Francisco,

CA

(2008) http://www.gene.com/gene/products/informati on/pdf/lucentis-prescribing.pdfAccessed June 10, 2008.

21. Yuan $A$, Ahmad BU, Xu D, Singh RP, Kaiser PK, Martin DF et al. Comparison of intravitreal ranibizumab and bevacizumab for the treatment of macular edema secondary to retinal vein occlusion. Int J Ophthalmol.2014;7(1):86-91

22. Iturralde D, Spaide RF, Meyerle CB, Klancnik JM, Tannuzzi LA, Fisher $Y L$ et al. Intravitreal bevacizumab (Avastin) treatment of macular edema in central retinal vein occlusion: a shortterm study. Retina.2006;26(3):279-84

23. Haller JA, Bandello F, Belfort R Jr, Blumenkranz MS, Gillies $M$, Heier J et al. OZURDEX GENEVA Study Group. Randomized, sham-controlled trial of dexamethasone intravitreal implant in patients with macular edema due to retinal vein occlusion. Ophthalmology. 2010;117(6):11341146.

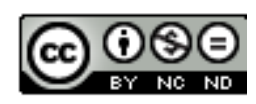

This work licensed under Creative Commons Attribution 